\title{
CROSSED CEREBELLAR DIASCHISIS AFTER STROKE: A VASCULAR PHENOMENON?
}

\section{Lita von Bieberstein*, Bas van Niftrik*, Martina Sebök², Marco Piccirelli³, Oliver Bozinov², Luca Regli², Andreas Luft ${ }^{1}$, Jorn Fierstra², Susanne Wegener ${ }^{1}$}

-1 Dept. of Neurology, Clinical Neuroscience Center, University Hospital Zurich and University of Zurich -2 Dept. of Neurosurgery, Clinical Neuroscience Center, University Hospital Zurich and University of Zurich -3 Dept. of Neuroradiology, Clinical Neuroscience Center, University Hospital Zurich

UniversitätsSpital Zürich

\section{Introduction}

Crossed cerebellar diaschisis (CCD) in internal carotid artery (ICA) territory stroke refers to a reduction in cerebral blood flow $(\mathrm{CBF})$ and energy metabolism on the contralateral cerebellar hemisphere. $(1,2,3)$ The term "diaschisis" was introduced by Monakow in 1914 (Greek dia "in half" and schizien "to split"), referring to a remote loss of excitability due to a focal brain lesion, induced by functional disconnection. (4) CCD can often be found in patients with stroke due to large vessel occlusion, however its influence on clinical outcome has been controversial. (5)

$C C D$ can be detected with $\mathrm{H}_{2} 0-\mathrm{PET}$, contemporary MRI techniques (DSC, ASL), and most recently with BOLD (blood oxygenation-level dependent) functional MRI. $(6,7)$ However, in patients with ICA occlusion, compensatory hemodynamic factors may also influence perfusion in remote brain areas, including the posterior circulation. (8) Patients with large vessel occlusion tend to have a higher risk of a recurrent ischemia if hemodynamic compensation is insufficient and cerebrovascular reactivity (CVR) is reduced (9). Our goal was to identify potential hemodynamic contributions of CCD in patients with ICA occlusion, using BOLD-CVR imaging and Transcranial Duplex sonography (TCD).

\section{Methods}

TCD measurement of flow in the MCA-M1, ACA, PCA and the presence of the four main collateral pathways $(8,9)$ as well as clinical data (NIHSS and mRS) from 19 stroke patients with unilateral ICA occlusion who underwent BOLD-MRI-CVR assessment were analyzed. The presence of CCD (CCD + or CCD -) was judged quantitatively from BOLD images, based on a cerebellar asymmetry Index cut-off value of $>+7 \%$ (that means that the CVR of the contralateral cerebellar hemisphere is on average $7 \%$ lower then the ipsilateral cerebellar hemisphere). Non-parametric tests were used for group comparisons between CCD+ and CCD - (significance level $p$ $<0.05)$.
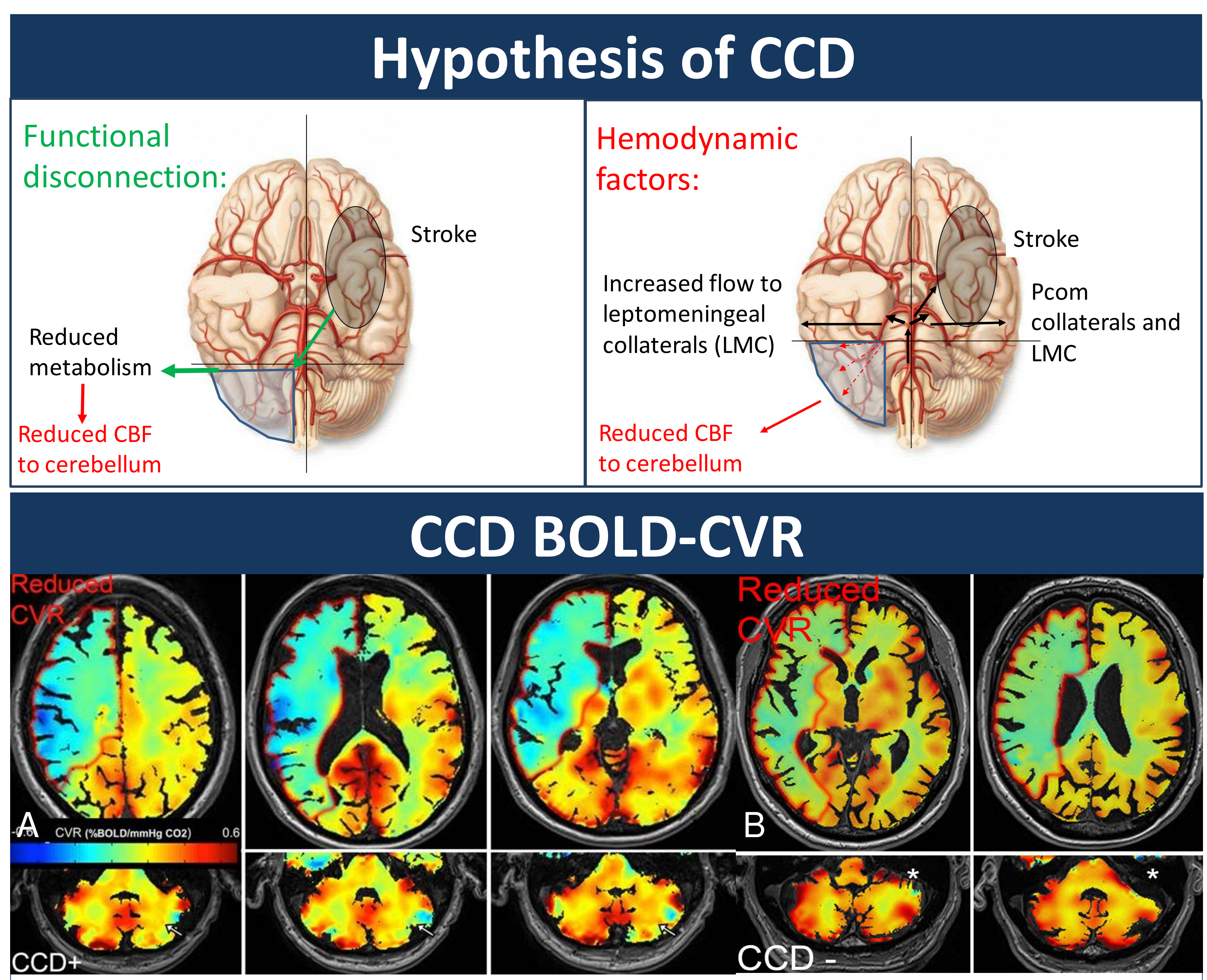

Demonstration of supra- and infratentorial BOLD-CVR in a patient with right ICA occlusion. Supratentorially there is reduced CVR of the right hemisphere.

(A) shows a CVR reduction of the contralateral cerebellar hemisphere (CCD +) (B) shows no CVR reduction of the contralateral cerebellar hemisphere (CCD -).

\section{Conclusions and Outlook}

In patients with stroke due to ICA occlusion, the presence of CCD was associated

1) more severe stroke deficits on admission,

2) higher flow in both P2 segments of the PCA and in the contralateral P1 segment

The latter is likely to indicate increased compensatory collateral recruitment through leptomeningeal collaterals in CCD+ patients. Our data suggest that in patients with ICA occlusion, there may be an additional hemodynamic contribution to CCD; indicating more severe hemodynamic compromise.

Recruitment of more patients as well as consideration of infarct size and location will yield additional information about this phenomenon.

\begin{tabular}{|c|c|c|c|}
\hline \multicolumn{4}{|c|}{ Results } \\
\hline $\mathrm{n}(\%)$ or median (IQR) & $C C D+n=10$ & $C C D-n=8$ & P value \\
\hline Age & $68.8(23)$ & $66.5(13)$ & 0.965 \\
\hline sex (female) & $3(33)$ & $2(25)$ & 0.814 \\
\hline NIHSS @ admission & $5(7)$ & $0(3)$ & 0.014 \\
\hline NIHSS@ 3 months & $1(1)$ & $0(0)$ & 0.193 \\
\hline mRS @ 3 months & $1(1)$ & $0(0)$ & 0.146 \\
\hline $\begin{array}{l}\text { CVR supratentorial } \\
\text { affected }\end{array}$ & $0.0609(0.1)$ & $0.0819(0.12)$ & 0.515 \\
\hline $\begin{array}{l}\text { CVR cerebellar } \\
\text { ipsilateral }\end{array}$ & $0.1825(0.17)$ & $0.1713(0.06)$ & 0.696 \\
\hline $\begin{array}{l}\text { CVR cerebellar } \\
\text { contralateral }\end{array}$ & $0.1420(0.18)$ & $0.1646(0.06)$ & 0.829 \\
\hline $\begin{array}{l}\text { Number of coll. } \\
\text { (duplex) }\end{array}$ & $2(1)$ & $2(0)$ & 0.315 \\
\hline Coll_OA & $9(90)$ & $6(75)$ & 0.396 \\
\hline Coll_ACA & $9(90)$ & $6(75)$ & 0.396 \\
\hline Coll_Pcom & $1(10)$ & $3(38)$ & 0.163 \\
\hline Coll_Lepto & $2(20)$ & $0(0)$ & 0.180 \\
\hline PSV M1 ipsi (cm/s) & $79.5(56)$ & $80.5(69)$ & 1 \\
\hline PSV M1 con & $103.5(48)$ & $110(21)$ & 0.633 \\
\hline PSV ACA ipsi & $70(32)$ & $72.5(65)$ & 0.633 \\
\hline PSV ACA con & $141.5(115)$ & $97(70)$ & 0.234 \\
\hline PSV P1 ipsi & $84(39)$ & $68.5(39)$ & 0.237 \\
\hline PSV P1 con & $85(50)$ & $49(25)$ & 0.002 \\
\hline PSV P2 ipsi & $76(42)$ & $50(23)$ & 0.003 \\
\hline PSV P2 con & $76(26)$ & 49 (18) & 0.004 \\
\hline
\end{tabular}

Table 1) Patient characteristics, clinical outcome parameters, CVR descriptive parameters, Collaterals (qualitative and quantitative) and peak systolic velocity (PSV) of the ispi- and contralateral A. cerebri media (M1), A. cerebri anterior (ACA), A. cerebri posterior (P1 Segment, P2-Segment)

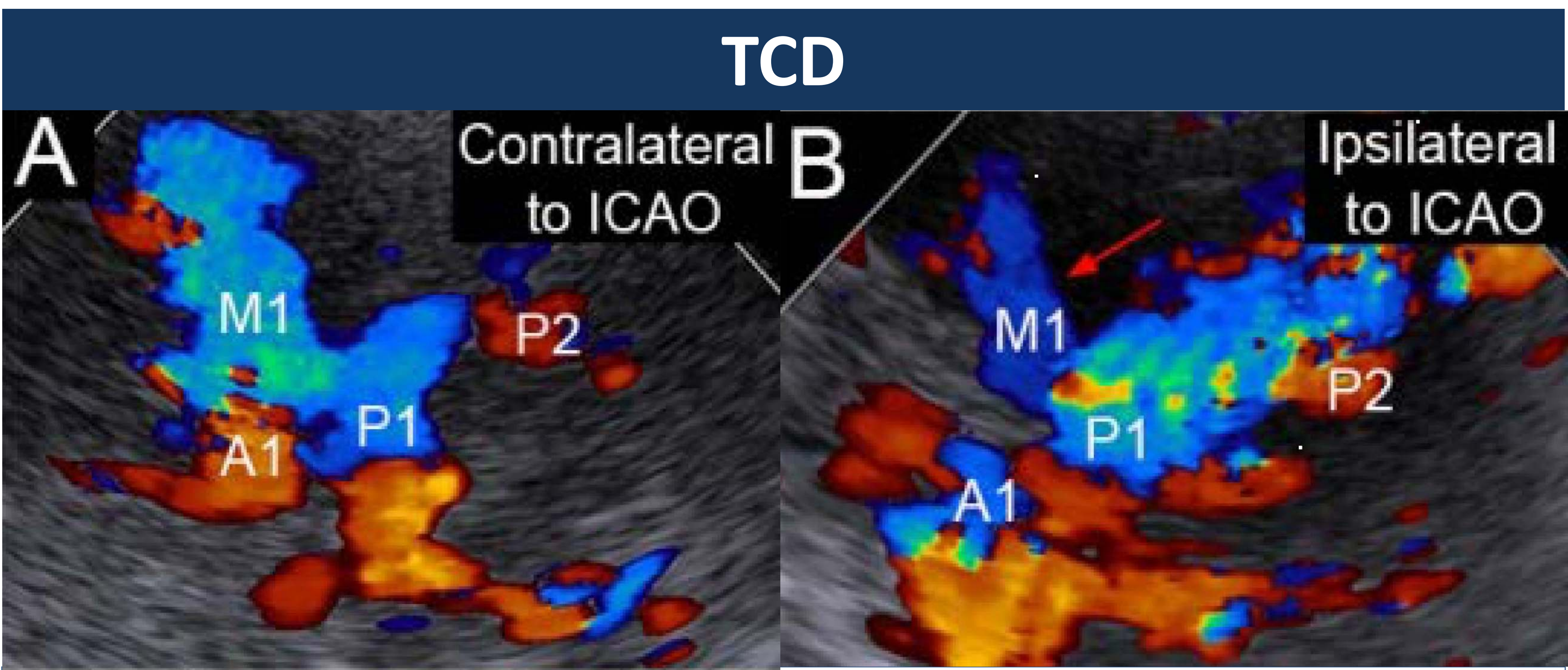

A) TCD contralateral to ICAO. Arterial segments: M1: MCA-M1, A1: ACA-A1, P1: PCA- P1, P2: PCA-P2.

B) TCD ipsilateral to ICAO. Same arterial segments and flow profiles as in A) on the affected side are shown. Flow direction in A1 is reversed (AcoA collateral).

Note: Flow values in both PCA are high. From Ref. (8).

1) Baron JC et al. Crossed cerebellar diaschisis in human supratentorial brain infarction. Trans Am Neurol. Assoc 1981

2) Baron JC et al. Local interrelationships of cerebral oxygen consumption and glucose utilization in normal subjects and in ischemic stroke patients: a positron tomography study. J Cereb Blood Flow Metab 1984;

3) YamucuchiH et al. Hemodynamic and metabolic changes in crossed cerebellar hypoperfusion. Stroke 1992;

4) Carrera and Tononi. Diaschi

Kunz et al., Crossed cerebellar diaschisis in acute ischemic stroke: Impact on morphologic and functional outcome JCBFM 2017

6) Fierstra et al Staging Hemodynamic Failure with Blood Oxygen-Level-Dependent Functional Magnetic Resonance Imaging Cerebrovascular Reactivity. Stroke 2018

Strother et al. Crossed cerebellar diaschisis after stroke identified nonivasively with cerebral blood flow-weighted arteria spin labelling MRI. Eur J Radiol, 2016)

8) Schneider et al., Ultrasound and Clinical Predictors of Recurrent Ischemica in Symptomatic Internal Carotid Artery Occlusion. Stroke 2015

Reinhard $\mathrm{M}$ et al. Dynamic cerebral autoregulation and collateral flow patterns in patients with severe carotid stenosis or occlusion. Ultrasound in medicine \& biology. 2003

10) Güler S. et al. Early clinical signs, lesion localization, and prognostic factors in unilateral symptomatic internal carotid artery occlusion. I Stroke Cerebrovasc Dis. 2014

Silvestrini et al Impaired cerebral vasoreactivity and risk of stroke in patients with asymptomatic interna cariotid artery stenosis JAMA. 20 